\title{
Characteristics of secondary slip fronts associated with slow earthquakes in Cascadia
}

\author{
Quentin Bletery $^{\mathrm{a}}$, Amanda M. Thomas ${ }^{\mathrm{a}}$, Jessica C. Hawthorne ${ }^{\mathrm{b}}$, Robert M. \\ Skarbek $^{\mathrm{c}}$, Alan W. Rempel ${ }^{\mathrm{a}}$, Randy D. Krogstad ${ }^{\mathrm{a}}$ \\ ${ }^{a}$ Department of Earth Sciences, University of Oregon, 1272 University of Oregon, \\ Eugene, Oregon 97403, USA. \\ ${ }^{b}$ University of Leeds, School of Earth and Environment, Leeds, United Kingdom. \\ ${ }^{c}$ Lamont-Doherty Earth Observatory, Columbia University, Palisades, New York, USA.
}

\section{Abstract}

We implement an algorithm to automatically detect migrations of low frequency earthquakes at time scales between $30 \mathrm{~min}$ and $32 \mathrm{~h}$ during the 2003, 2004 and 2005 slow slip events in Cascadia. We interpret these migrations of seismicity as a passive manifestation of secondary slip fronts (SSFs) that propagate faster than the main front. We identify the dominant features of 383 SSFs, including time, location, duration, area, propagation velocity and estimate: their moment, stress drop, slip, and slip rate. We apply the same algorithm to continuous tremor detection in Cascadia between 2009 and 2015 and characterize 693 SSFs at time scales between $4 \mathrm{~h}$ and $32 \mathrm{~h}$. We identify — to our knowledge for the first time - numerous 10-24 h long SSFs that propagate at velocities intermediate between slow slip events and previously reported SSFs. The systematic detection of SSFs fills a gap between seismically and geodetically detectable slow earthquake processes. Analyses of SSF basic features indicates a wide range of stress drops and slip rates (with me-

Email address: qbletery@uoregon.edu (Quentin Bletery)

Preprint submitted to Earth and Planetary Science Letters

January 30, 2017

(C) 2017. This manuscript version is made available under the Elsevier user license http://www.elsevier.com/open-access/userlicense/1.0/ 
dians of $5.8 \mathrm{kPa}$ and $1.1 \mathrm{~mm} / \mathrm{h}$ ) as well as an intriguing relationship between SSF direction and duration that was observed in other contexts and could potentially help discriminate between the different physical models proposed to explain slow slip phenomena.

Keywords: Slow slip, low-frequency earthquakes, nonvolcanic tremor, secondary slip fronts, Cascadia

\section{1. Introduction}

2 Slow slip events (SSEs) in subduction zones propagate along the plate 3 interface at velocities of 5-10 km/day and are largely confined to the region 4 known as the transition zone, located down-dip of the seismogenically locked 5 zone (Dragert et al., 2001; Bartlow et al., 2011). As SSEs propagate, small on-

6 fault asperities capable of generating seismic radiation fail in earthquake-like 7 events known as low-frequency earthquakes (LFEs) and nonvolcanic tremor 8 (Obara, 2002; Kao et al., 2006; Ide, 2010; Ueno et al., 2010; Houston et al., 9 2011). Multiple lines of evidence indicate that LFEs and tremor - which has

been interpreted as a superposition of LFEs (Shelly et al., 2007b) - are a passive manifestation of surrounding aseismic slow slip (Shelly et al., 2007b). The occurence of LFEs and tremor during SSEs are not random. Instead, spatially and temporally coherent migrations of LFEs and tremor can often be identified within the actively slipping portion of the fault after the main slip front associated with the SSE has passed. These migration patterns are thought to represent secondary slip fronts (SSFs) occurring over several different length and timescales. For example, Houston et al. (2011) and Royer et al. (2015) used tremor and LFEs to image approximately $40 \mathrm{~km}$ long slip 
fronts - known as rapid tremor reversals (RTRs) — that propagate back in the opposite direction but 10 to 40 times faster than the main slip front. Ghosh et al. (2010) used tremor in Cascadia to image seismic migrations — known as streaks — that propagate back and forth along the main slip front (i.e., perpendicular to its propagation direction) but approximately 100 times faster. Rubin and Armbruster (2013) reported tremor migrations at the kilometer scale that propagate along strike either towards or away from the main front, but approximately ten times faster. Finally, Peng et al. (2015a) and Peng and Rubin (2016) found evidence for complex tremor migration patterns beneath that evolve as the main front propagates. While all of the aforementioned studies were conducted in Cascadia, similar observations have been reported in Japan (Shelly et al., 2007a; Ueno et al., 2010; Obara et al., 2012), Mexico (Radiguet et al., 2012; Frank et al., 2014; Peng and Rubin, 2017) and along the San Andreas fault (Ryberg et al., 2010; Shelly, 2009, 2010; Peng et al., 2015b), suggesting that SSFs may be a ubiquitous feature of slow slip.

Slow-slip phenomena require the fault slip rate to increase to observed speeds, typically 10 to 100 times the plate rate, but abstain from accelerating to typical earthquake slip rates. The specific physical mechanisms responsible for imposing this speed limit are still debated. SSFs generally propagate at least an order of magnitude faster than the main front associated with the SSE, and estimates of the ratio of the SSF stress drop to that of the main front range between one tenth and one (Rubin and Armbruster, 2013; Royer et al., 2015; Hawthorne et al., 2016). These stress drop estimates combined with the propagation speeds mentioned above imply that SSFs must be slipping either 
as fast as or faster than the main front. Hence, reproducing stress drops, slip and propagation speeds of both the main and secondary fronts requires a rheology that limits the speed of the main front while allowing the SSFs to slip one to two orders of magnitude faster. Such behavior is difficult to reproduce with a single state variable friction law (Rubin, 2011). Additionally, SSFs appear to be sensitive to very small $(\approx 1 \mathrm{kPa})$ tidal stress perturbations (Thomas et al., 2013; Royer et al., 2015; Houston, 2015) allowing them to be used to probe in situ conditions in deep faults. Observations of SSFs, in addition to SSEs, may provide useful constraints on physical models of slow slip processes (Rubin, 2011; Hawthorne and Rubin, 2013).

To date, most studies that have documented SSFs have relied on subjective identification methods such as visual identification, often using only one dimension of space and time (Shelly et al., 2007a; Ueno et al., 2010; Ghosh et al., 2010; Houston et al., 2011; Rubin and Armbruster, 2013; Royer et al., 2015; Peng et al., 2015a; Savard and Bostock, 2015). While these approaches successfully find some SSFs, such subjective identification makes it possible and even likely that many SSFs go undetected. Here we develop an algorithm that systematically identifies SSFs based on spatial and temporal migration of tremor/LFEs and discuss its advantages compared to the similar method developed by Obara et al. (2012). We apply our algorithm to both tremor and LFE datasets resulting from SSEs that occurred between 2003 and 2015 in Cascadia. We detect over 1000 SSFs, determine their locations, duration, directions, and estimate some of their physical properties, such as moment, average slip, stress drop and and slip rate. 


\section{Data and algorithm}

We use two types of data: a catalog of LFEs located near Vancouver Island during the 2003, 2004 and 2005 SSEs (Savard and Bostock, 2015) and a catalog of continuous tremor detections from August 2009 to December 2015 that occur throughout the Cascadia subduction zone (Wech and Creager, 2008; Wech, 2010). The LFE catalog was obtained using cross-station waveform correlation; thanks to a dense campaign seismic network, it has very good spatial (location errors of $<1 \mathrm{~km}$ ) and temporal resolution (Savard and Bostock, 2015). The tremor catalog contains locations and detection times of tremor events determined using waveform envelope correlation and clustering (Wech and Creager, 2008; Wech, 2010). Detections correspond to 5-minute seismogram windows that likely represent a superposition of LFEs (Shelly et al., 2007b). Therefore, resolution of both time (each detection is separated by at least $2.5 \mathrm{~min})$ and location $(5-10 \mathrm{~km})$ is worse than for the LFE catalog. Because the tremor detection algorithm is independently implemented on several adjacent seismic networks, some tremor detections appear twice in the final catalog (Wech, 2010). To eliminate duplicate detections, when two tremors are detected at the same time within $25 \mathrm{~km}$, we exclude one (Wech, 2010).

These two catalogs provide a large number of identified LFE and tremor events. To identify possible SSFs, we analyze LFE and tremor migration patterns in 3 dimensions: distance along strike, distance along dip, and time. The algorithm is illustrated in Figure 1. As in the approach of Obara et al. (2012), we look for SSFs in time windows of various durations. The different time windows considered in our analysis are 30 min, 1 h, 2 h, 4 h, 8 h, 16 h, 
$32 \mathrm{~h}$ for the LFE catalog and $4 \mathrm{~h}, 8 \mathrm{~h}, 16 \mathrm{~h}, 32 \mathrm{~h}$ for the tremor catalog. As individual detections are all separated by at least $2.5 \mathrm{~min}$ (Wech and Creager, 2008), the number of potential detections in a $\leq 2 \mathrm{~h}$ time window too small to analyze their potential migration. The analysis is performed independently on the different time windows. Therefore, tremor/LFE detections may theoretically be part of more than one SSF in different time windows; thus a short duration SSF may be a part of a longer duration SSF.

We first identify clusters of LFE and tremor detections in the individual catalogs. We use the subclust matlab routine to identify LFE/tremor clusters of different duration. The subclust routine estimates the centers of subclusters of a given duration in a data set using subtractive clustering (Chiu, 1994; Yager and Filev, 1994). We set the duration of the subclusters to correspond to the different time windows. The subclust routine then returns the tremor/LFE clusters of $30 \mathrm{~min}$ to $32 \mathrm{~h}$ duration with the highest concentration of events. For each identified cluster, we then calculate the linear regression of the distance along strike as a function of time and exclude points farther than two standard deviations $(2 \sigma)$ from the regression line. After removing those points, we do the same linear regression but for the distance along dip as a function of time and remove events farther than $2 \sigma$ from the linear regression. Finally, we project the remaining points along the best-fitting migration axis and remove again events that lie outside $2 \sigma$ from the linear regression of the distance along the migration axis as a function of time. The remaining points are then tested: if the cluster still contains more than $20 \mathrm{LFE}$ or tremor detections and if the root mean square (RMS) of their distance to the last regression is smaller than $15 \%$ of the migration 
length, then the cluster is identified as an SSF. For a given time window, if the SSF length is small, then the front is not moving quickly (the signal is low). Thus we require the deviation from the propagation axis (the noise) to be very low (to ensure a decent signal to noise ratio). If the SSF length is larger, then the front is clearly moving (the signal is larger) and we can be more tolerant with the deviation from the propagation axis (as we have a clear signal). We repeat the process for all the clusters. We then remove the LFE or tremor detections identified as part of an SSF from the catalog and rerun the clustering routine on the new catalog with identified SSFs removed. We iterate until the catalog no longer contains any LFE or tremor. If no SSF is identified on one iteration, we remove all the tested clusters from the catalog and iterate once more in the truncated catalog. All the detected SSFs are then tested to ensure the continuity of the migration. We subdivide the successfully identified SSFs into 4 equal portions along their best-fitting migration axis and 3 equal periods and if one of the parts or periods contains less than $1 / 20$ th of the number of LFE/tremor detections that compose the $\mathrm{SSF}$, the SSF is rejected in order to ensure that each SSF is continuous rather than composed of two distinct SSFs.

Our approach is inspired by Obara et al. (2012) (who implemented an automatic SSF detection based on principal component analysis of tremor in Japan), but has some significant differences. Obara et al. (2012) identified SSFs using moving time windows shifted by discrete increments. As it is unlikely that SSFs all start and end at the specific times of arbitrary time windows, this may result in detecting SSFs that either are just a part of a larger undetected SSFs or include LFE/tremor that should not be associated 
with the SSF, potentially affecting the apparent migration velocities. The use of a clustering subroutine allows for flexibility in the start and end time of the explored SSFs - as tested time windows are not shifted by discrete increments but determined in respect of high concentrations of tremor/LFEs — substantially increasing the number of SSF detections. Another significant difference is the detection thresholds. In the work of Obara et al. (2012), SSF acceptance is based on linearity, angular difference and in the number of tremor detections, all of which vary with the given time window. We apply only two thresholds, which are the same for all time windows: the number of LFE/tremor detections (20 for all time windows) and a deviation criteria based on the ratio of the misfit to the SSF length (required to be $\leq 15 \%$ ), which can be interpreted as a signal to noise ratio. Our choices of $20 \mathrm{LFE} /$ tremor detections and $15 \%$ are arbitrary — as are the thresholds used by Obara et al. (2012) - but these values do provide visually coherent patterns in all the detected SSFs (Figures 2, 3).

To ensure that our algorithm does not interpret noise as SSF, we test it on synthetic catalogs. To produce the synthetic catalogs, we use the actual catalog locations and randomize the time of occurrence. We run the algorithm for all the time windows of our analysis and detect no SSF in the synthetic catalogs.

\section{Spatial distribution of secondary slip fronts}

We identified 383 SSFs in the LFE catalog of the 2003, 2004, and 2005 SSEs beneath Vancouver Island and 693 SSFs in the tremor catalog between August 2009 and December 2015 in Cascadia. While SSFs lasting from 10 
min to $11 \mathrm{~h}$ have been documented in multiple subduction zones (Ghosh et al., 2010; Houston et al., 2011; Obara et al., 2012; Rubin and Armbruster, 2013; Royer et al., 2015; Peng et al., 2015a; Savard and Bostock, 2015), we identified - to our knowledge for the first time - numerous SSFs lasting between 11 and $22.5 \mathrm{~h}$ in both catalogs (243 in the tremor catalog, 57 in LFEs catalog). They propagate at intermediate speeds $(\approx 3 \mathrm{~km} / \mathrm{h})$ between those of the main front $(\approx 0.2-0.5 \mathrm{~km} / \mathrm{h})$ and previously documented SSFs ( $\approx 40 \mathrm{~km} / \mathrm{h}$ ) (Ghosh et al., 2010; Houston et al., 2011; Obara et al., 2012; Rubin and Armbruster, 2013; Royer et al., 2015; Peng et al., 2015a; Savard and Bostock, 2015).

The spatial distribution of SSFs is of particular interest because it can reveal atypical behaviors of some fault areas and may highlight spatial variations of fault properties. Figure 4 shows the distribution of tremor derived SSFs during the 2009-2015 period along the Cascadia megathrust by time window. Note that some long duration tremor derived SSFs may correspond to the main front of short duration SSEs. In the inset diagrams we plot the number of SSFs as a function of their propagation directions. Some areas show a clear tendency to host numerous SSFs (for instance at latitude $40^{\circ} \mathrm{N}-41.5^{\circ} \mathrm{N}, 42^{\circ} \mathrm{N}-42.5^{\circ} \mathrm{N}$, or $44.5^{\circ} \mathrm{N}-45.5^{\circ} \mathrm{N}$ ) whereas other regions had no detectable SSFs. Additionally, there is a clear preference for along-strike propagation for the majority of the tremor derived SSFs. Both the spatial distribution and propagation directions are consistent from SSE to SSE, suggesting that the physical mechanism responsible for generating SSFs does not change over a timescale of several years.

Because both the temporal and spatial resolution are much better in the 
LFE catalog, we are able to detect short timescale SSFs beneath Vancouver island (Figure 5). Since the 3 SSEs that drove LFEs in this area all roughly propagated in the same direction (blue arrow in Figure 5), this catalog is also more convenient to analyze the direction of SSFs with respect to the direction of SSEs. SSFs propagate in all directions and encompass phenomena previously described as RTRs or streaks. They also encompass slip fronts that propagate in the direction of the SSE (Figure 5). Such forward-propagating SSFs are hard to distinguish by eye from LFE or tremor migration associated with the main front. They typically initiate after the main front has passed and migrate towards the tip of the main front. Interestingly, we observe a clear relationship between the direction and the duration of SSFs. The vast majority of short duration (i.e. $30 \mathrm{~min}$ and $1 \mathrm{~h}$ ) SSFs propagate along dip. As the SSF duration increases, the proportion of SSFs propagating along strike gradually increases (see Rose diagrams in Figure 5) resulting in the longest duration LFE derived SSFs propagating almost solely along strike. Similar relationships were observed by Ghosh et al. (2010) and Houston et al. (2011) in Cascadia and by Obara et al. (2012) beneath the Kii peninsula in the Nankai subduction zone. Potential physical mechanisms responsible for SSFs will have to explain this enigmatic dependence between duration and direction (Rubin, 2011). The ratio of along-strike SSFs propagating in the SSE direction to along-strike SSFs propagating in the opposite direction also seems to depend on the duration. While reversal SSFs are predominant in the $8 \mathrm{~h}$ time window, almost all SSFs propagate forward in the $32 \mathrm{~h}$ time window.

The distribution of propagation velocity $V_{\text {prop }}$ is shown at the bottom of 
each subfigure in Figures 4 and 5. In individual time windows, along-dip SSFs do not appear faster than along-strike SSFs. However, as the length $L$ of SSFs is limited by the extent of the fault, the average propagation velocity decreases with the duration $T$ (because $V_{\text {prop }}=L / T$ ). Accordingly, the limited number of distinct time windows in the analysis of the tremor catalog results in little difference in the propagation velocity (Figure S1.f).

\section{Physical characteristics of secondary slip fronts}

Our detection algorithm provides a number of direct observations characterizing each SSF including start time, location, direction (Figures 4, 5), duration $T$, length $L$, width $W$, pulse (i.e. actively slipping) length $l$ (Figure 6), propagation velocity $V_{\text {prop }}$ (defined as $L / T$ ), and number of LFE or tremor detections $n_{e}$. The distributions of these SSF properties are shown in Figure S1 (catalogs are available in the supplementary materials). Assessing other physical characteristics of SSFs, such as moment or stress drop, is challenging because these quantities require an estimate of the total slip associated with each SSF.

Individual SSFs are too small to generate a surface deformation signal detectable with GPS and hence most studies that have estimated slip for individual SSFs have relied on the total SSE moment and the fraction of LFE detections or bursts that occured as part of the SSF (Rubin and Armbruster, 2013; Royer et al., 2015). Here, we adopt a similar approach and assume that the total moment $M_{e}$ associated with each LFE or tremor detection is the same (although different for LFEs and tremor). This assumption is demonstrably false for individual LFE repeats, which have been shown to 
have very different moments (Bostock et al., 2015), and for individual tremor events, as their definition (i.e. the 2.5 minute timescale used to separate individual events) is arbitrary and includes no contribution from waveform amplitudes (Wech and Creager, 2008). However, since moment estimates for each SSF include a minimum of 20 LFEs or tremors, it is likely that the average moment will be a more accurate measure of total SSF moment. Nevertheless, our estimates of the physical characteristic of SSFs (such as moment, slip, stress drop, slip rate) are tentative and caution should be exercised when interpreting them.

We obtain $M_{e}$, the average moment associated with each LFE or tremor, by dividing the moment $M_{0}$ (assessed by geodetic studies (Schmidt and Gao, 2010; Krogstad and Schmidt, 2015)) associated with an SSE by the number of events $N_{e}$ detected during the event:

$$
M_{e}=\frac{M_{0}}{N_{e}}
$$

An implicit assumption behind equation (1) is that moment associated with aseismic slip that does not generate LFE or tremor detections is negligible. The moment, $M_{S S F}$, associated with one SSF, is defined as the sum of the moment associated with all the LFE or tremor detections that are part of that SSF,

$$
M_{S S F}=\sum_{i=1}^{n_{e}} M_{e}(i),
$$

where $n_{e}$ is the number of $\mathrm{LFE} /$ tremor detections part of the given SSF. As we assumed that for all $i, M_{e}(i)=\frac{M_{0}}{N_{e}}$,

$$
M_{S S F}=\frac{n_{e}}{N_{e}} M_{0}
$$


Note that possible deviations from constant $M_{e}(i)$ would tend to be averaged out over the number of events in equation (3) because $M_{e}(i)$ is sometimes larger and sometimes smaller than the assumed average $M_{e}=\frac{M_{0}}{N_{e}}$. We further estimate an average slip $d$ for each SSF,

$$
d=\frac{M_{S S F}}{\mu A},
$$

where $\mu$ is the shear modulus and $A=W L$ is the SSF slip area, with the SSF width $W$ and length $L$ both estimated from our analysis (Figure 6). To be consistent with geodetic inversions, we use $\mu=40 \mathrm{GPa}$ (Schmidt and Gao, 2010; Krogstad and Schmidt, 2015). The stress drop $\Delta \sigma$ is then given by

$$
\Delta \sigma=\frac{4(\lambda+\mu)}{\pi(\lambda+2 \mu)} \mu \frac{d}{W}
$$

where $\lambda$ is Lamé's parameter (Lay and Wallace, 1995), which we set to $\lambda=40$ GPa. The total slip $d$ accumulates in the time the front propagates the distance $l$. Thus, we can deduce the slip rate $V_{\text {slip }}$ from the propagation velocity $V_{\text {prop }}$ as

$$
V_{\text {slip }}=\frac{d}{l} V_{\text {prop }}
$$

The distributions of assessed physical characteristics (moment, slip, stress drop and slip rate) are shown in Figures S2 and S3 (catalogs in the supplementary materials). Because we did not estimate these parameters independently, their distributions are correlated with each other (Figure S4). However, most of the correlation coefficients between the different parameters are relatively weak (i.e. below 0.6 in magnitude, see Figure S4). As expected, high correlation coefficients are found for slip and stress drop, since the stress drop is estimated from slip (eq. 5), and between the different spatial dimensions $(L, W$ and $l$ ), implying that the aspect ratios of SSFs do not vary 
appreciably. Significant anticorrelations are observed between propagation rate $V_{\text {prop }}$ and duration $T$, as well as between the spatial dimensions and both slip $d$ and slip rate $V_{\text {slip }}$. It is also noteworthy that stress drop $\Delta \sigma$ appears to be independent of moment $M$ in both catalogs (Figure S4).

The median stress drop among all SSFs is $5.8 \mathrm{kPa}$, which is very consistent with stress drop estimates obtained for RTRs using strainmeters (8 $\mathrm{kPa}$ ) (Hawthorne et al., 2016). These values of stress drop are several times smaller than typical stress drops in SSEs $(10$ - $100 \mathrm{kPa})$, and over 2 orders of magnitude smaller than regular earthquakes (Gao et al., 2012). The estimated stress drops are also all consistently smaller than effective normal stress (0.2-3 MPa) inferred in LFE source regions (Liu and Rice, 2007; Hawthorne and Rubin, 2013; Royer et al., 2015). The median slip is $3 \mathrm{~mm}$ and the median slip rate is $1.1 \mathrm{~mm} / \mathrm{h}$, which is much faster than slip rates inferred for SSEs (on the order of 1-2 mm/day (Wech and Bartlow, 2014)). The observation that SSF slip rates are significantly faster than SSE slip rates is consistent with previous studies (Rubin and Armbruster, 2013; Royer et al., 2015; Hawthorne et al., 2016) and implies that the fault rheology limits the main front speed and then allows secondary fronts to slip faster. As acknowledged in the introduction, such a time dependent behavior is not easy to reproduce with a single state variable friction law (Rubin, 2011). SSF slip rates may then be used to constrain physical models of slow slip phenomena.

Though variations in the moments and slip distributions of SSFs range within a single order of magnitude $\left(4.10^{16}\right.$ to $4.10^{17}$ N.m and 0.1 to $\left.40 \mathrm{~mm}\right)$ for all SSFs, stress drop varies by over 3 orders of magnitude (from 0.1 to $300 \mathrm{kPa}$ ), as does slip rate (between 0.02 and $30 \mathrm{~mm} / \mathrm{h}$ ) (Figures $\mathrm{S} 2$ and 
S3). We recall here that our estimates of stress drop are based on the original assumption that SSF moment scales with the number of LFE/tremor detections, which could be inaccurate for some SSFs. Large scatter in slip, stress drop and slip rate is also expected from equations (4), (5) and (6) as a result of multiplying quantities $\left(M_{S S F}, L, W, l\right.$ and $\left.V_{\text {prop }}\right)$ that have large variability. Therefore a large part of the scatter in slip rate and stress drop might be attributed to assumption inaccuracy and propagation of uncertainties, but some of this scatter could also be sourced to changes in fault properties. As SSF areas widely overlap - this is particularly clear in the LFE catalog (Figure 5) - the varying SSF properties might suggest that, in addition to being able to slip at different rates, the same fault area is also able to release very different amounts of stress.

\section{Moment - duration scaling}

Ide et al. (2007) suggested that slow slip phenomena - including LFEs, very low frequency earthquakes (VLFEs) and SSEs - follow a moment duration scaling law distinct from regular earthquakes. While regular (fast) earthquakes have moment proportional to the cube of their duration, slow slip phenomena would have moment proportional to duration (with $M / T=10^{12}$ $10^{13} \mathrm{~N} . \mathrm{m} / \mathrm{s}$ ) (Figure 7). In this representation, important gaps separate the various slow slip phenomena (Ide et al., 2007). The question of whether these gaps result from detection limitations or from the inherent behavior of fault slip is of importance because it could provide us with physical constraints on earthquake processes (Ide et al., 2007; Ide, 2008; Ide et al., 2008; Peng and Gomberg, 2010; Gao et al., 2012; Ide and Yabe, 2014; Liu, 2014; Gomberg 
et al., 2016).

In the far field, seismic amplitudes scale with moment rate (Aki and Richards, 2002). Thus, the threshold for seismically detectable signals follows the noise level in the different frequency bands. We use the USGS low noise model (Peterson et al., 1993; Ide, 2014) and empirically calibrate it on the smallest moment rate events that have been seismically detected (VLFEs from Ide and Yabe (2014)) to determine a threshold for seismically detectable events (red dashed line in Figure 7). Existing geodetic instrumentation has proven capable of detecting magnitude 5.7-6.0 events and above regardless of their duration (Peng and Gomberg, 2010) (blue dashed line in Figure 7). The gap between LFEs and VLFEs (Figure 7) may be attributed to particularly large noise in the frequency band corresponding to the gap (Ide et al., 2008) while the gap between the VLFEs and SSEs corresponds to moments too low to be detected by geodesy (Peng and Gomberg, 2010) and characteristic periods too long to be detected in seismograms (Ide et al., 2008).

The large range of duration and moment among the SSFs we detected follows the slow slip scaling law and fills the gap between VLFEs and SSEs, where signal is not directly detectable either from seismic or geodetic records (Figure 7). The vertical (moment) threshold in SSFs corresponds to the minimum number of LFE/tremor detections (20) in one SSF times $M_{e}$ and the distinct horizontal lines in SSF duration correspond to our different time windows. These two noncontinuous characteristics are consequences of our approach. Interestingly, SSFs appear to follow the same threshold of constant moment rate - on the order of $10^{13} \mathrm{~N} . \mathrm{m} / \mathrm{s}$ - as the other slow slip phenomena (Figure 7). The fact that SSFs follow the slow slip phenomena 
scaling law may be a consequence of estimating SSF moment from moment of SSEs (eq. 3), as SSEs follow this law. Nevertheless, it is worth noting that our moment estimates are in agreement with SSF moments estimated from strain meters (Hawthorne et al., 2016) (green square in Figure 7).

While individual slow slip phenomena do not appear to have moment proportional to duration (e.g. Ide et al. (2008); Bostock et al. (2015); Gomberg et al. (2016)), they all fit a constant moment rate scaling law (within less than 2 orders of magnitude variation) when considered together on a 10 orders of magnitude scale (Figure 7). More generally, fast and slow slip phenomena appear to be well distinguished in Figure 7. Fault slip processes that would range between the two scaling laws are, in theory, detectable - and should therefore have been detected - by seismology, geodesy or both (Figure 7). The large gap in detection of fault slip processes between the two scaling laws suggests that earthquakes and slow slip phenomena are two very distinct fault slip processes.

\section{Conclusion}

We identified a total of 1076 SSFs in Cascadia at timescales between 30 min and $32 \mathrm{~h}$. We cataloged their duration, width, length, pulse length and time of occurrence and provided estimates for some of their physical properties such as moment, slip, slip rate, and stress drop. The median moment is $5.3 \times 10^{16} \mathrm{~N} . \mathrm{m}$ (corresponding to a moment magnitude of 5.1), the median slip is $3 \mathrm{~mm}$, the median slip rate is $1.1 \mathrm{~mm} / \mathrm{h}$ and the median stress drop is $5.8 \mathrm{kPa}$. Moment estimates suggest that SSFs bridge a major gap between seismically and geodetically detectable events in the slow slip phe- 


\section{Acknowledgments}

This work was funded by the NSF grant EAR-1520238. The tremor catalog was made available online by A. Wech and K. Creager (tunk.ess.washington.edu/map_display/). We thank G. Savard and M. Bostock for providing the LFE catalog.

\section{References}

Aki, K., Richards, P. G., 2002. Quantitative seismology. Vol. 1.

Bartlow, N. M., Miyazaki, S., Bradley, A. M., Segall, P., 2011. Space-time correlation of slip and tremor during the 2009 cascadia slow slip event. Geophysical Research Letters 38 (18).

Bletery, Q., Sladen, A., Delouis, B., Vallée, M., Nocquet, J.-M., Rolland, L., Jiang, J., 2014. A detailed source model for the mw9. 0 tohoku-oki earthquake reconciling geodesy, seismology, and tsunami records. Journal of Geophysical Research: Solid Earth 119 (10), 7636-7653. 
Bletery, Q., Sladen, A., Jiang, J., Simons, M., 2016. A bayesian source model for the 2004 great sumatra-andaman earthquake. Journal of Geophysical Research: Solid Earth 121 (7), 5116-5135.

Bostock, M., Thomas, A., Savard, G., Chuang, L., Rubin, A., 2015. Magnitudes and moment-duration scaling of low-frequency earthquakes beneath southern vancouver island. Journal of Geophysical Research: Solid Earth $120(9), 6329-6350$.

Chiu, S. L., 1994. Fuzzy model identification based on cluster estimation. Journal of Intelligent \& fuzzy systems 2 (3), 267-278.

Delouis, B., Nocquet, J.-M., Vallée, M., 2010. Slip distribution of the february 27, $2010 \mathrm{mw}=8.8$ maule earthquake, central chile, from static and high-rate gps, insar, and broadband teleseismic data. Geophysical Research Letters 37 (17).

Dragert, H., Wang, K., James, T. S., 2001. A silent slip event on the deeper cascadia subduction interface. Science 292 (5521), 1525-1528.

Frank, W. B., Shapiro, N. M., Husker, A. L., Kostoglodov, V., Romanenko, A., Campillo, M., 2014. Using systematically characterized low-frequency earthquakes as a fault probe in guerrero, mexico. Journal of Geophysical Research: Solid Earth 119 (10), 7686-7700.

Gao, H., Schmidt, D. A., Weldon, R. J., 2012. Scaling relationships of source parameters for slow slip events. Bulletin of the Seismological Society of America 102 (1), 352-360. 
Ghosh, A., Vidale, J. E., Sweet, J. R., Creager, K. C., Wech, A. G., Houston, H., Brodsky, E. E., 2010. Rapid, continuous streaking of tremor in cascadia. Geochemistry, Geophysics, Geosystems 11 (12).

Gomberg, J., Wech, A., Creager, K., Obara, K., Agnew, D., 2016. Reconsidering earthquake scaling. Geophysical Research Letters 43 (12), 6243-6251.

Hawthorne, J., Rubin, A., 2013. Tidal modulation and back-propagating fronts in slow slip events simulated with a velocity-weakening to velocitystrengthening friction law. Journal of Geophysical Research: Solid Earth $118(3), 1216-1239$.

Hawthorne, J. C., Bostock, M. G., Royer, A. A., Thomas, A. M., 2016. Variations in slow slip moment rate associated with rapid tremor reversals in cascadia. Geochemistry, Geophysics, Geosystems, in review.

Houston, H., 2015. Low friction and fault weakening revealed by rising sensitivity of tremor to tidal stress. Nature Geoscience 8 (5), 409-415.

Houston, H., Delbridge, B. G., Wech, A. G., Creager, K. C., 2011. Rapid tremor reversals in cascadia generated by a weakened plate interface. Nature Geoscience 4 (6), 404-409.

Ide, S., 2008. A brownian walk model for slow earthquakes. Geophysical Research Letters 35 (17).

Ide, S., 2010. Striations, duration, migration and tidal response in deep tremor. Nature 466 (7304), 356-359. 
Ide, S., 2014. Modeling fast and slow earthquakes at various scales. Proceedings of the Japan Academy, Series B 90 (8), 259-277.

Ide, S., Beroza, G. C., Shelly, D. R., Uchide, T., 2007. A scaling law for slow earthquakes. Nature 447 (7140), 76-79.

Ide, S., Imanishi, K., Yoshida, Y., Beroza, G. C., Shelly, D. R., 2008. Bridging the gap between seismically and geodetically detected slow earthquakes. Geophysical Research Letters 35 (10).

Ide, S., Yabe, S., 2014. Universality of slow earthquakes in the very low frequency band. Geophysical Research Letters 41 (8), 2786-2793.

Ito, Y., Obara, K., 2006. Very low frequency earthquakes within accretionary prisms are very low stress-drop earthquakes. Geophysical Research Letters $33(9)$.

Kao, H., Shan, S.-J., Dragert, H., Rogers, G., Cassidy, J. F., Wang, K., James, T. S., Ramachandran, K., 2006. Spatial-temporal patterns of seismic tremors in northern cascadia. Journal of Geophysical Research: Solid Earth 111 (B3).

Krogstad, R. D., Schmidt, D. A., 2015. Assessing the updip spatial offset of tremor and slip during ets events in cascadia. In: 2015 AGU Fall Meeting. Agu.

Lay, T., Wallace, T. C., 1995. Modern global seismology. Vol. 58. Academic press. 
Liu, Y., 2014. Source scaling relations and along-strike segmentation of slow slip events in a 3-d subduction fault model. Journal of Geophysical Research: Solid Earth 119 (8), 6512-6533.

Liu, Y., Rice, J. R., 2007. Spontaneous and triggered aseismic deformation transients in a subduction fault model. Journal of Geophysical Research: Solid Earth 112 (B9).

Matsuzawa, T., Obara, K., Maeda, T., 2009. Source duration of deep very low frequency earthquakes in western shikoku, japan. Journal of Geophysical Research: Solid Earth 114 (B11).

Obara, K., 2002. Nonvolcanic deep tremor associated with subduction in southwest japan. Science 296 (5573), 1679-1681.

Obara, K., Matsuzawa, T., Tanaka, S., Maeda, T., 2012. Depth-dependent mode of tremor migration beneath kii peninsula, nankai subduction zone. Geophysical Research Letters 39 (10).

Peng, Y., Rubin, A. M., 2016. High-resolution images of tremor migrations beneath the olympic peninsula from stacked array of arrays seismic data. Geochemistry, Geophysics, Geosystems 17, 587-601.

Peng, Y., Rubin, A. M., 2017. Intermittent tremor migrations beneath guerrero, mexico and implications for fault healing within the slow slip zone. Geophysical Research Letters.

Peng, Y., Rubin, A. M., Bostock, M. G., Armbruster, J. G., 2015a. Highresolution imaging of rapid tremor migrations beneath southern vancou- 
ver island using cross-station cross correlations. Journal of Geophysical Research: Solid Earth 120 (6), 4317-4332.

Peng, Z., Gomberg, J., 2010. An integrated perspective of the continuum between earthquakes and slow-slip phenomena. Nature Geoscience 3 (9), 599-607.

Peng, Z., Shelly, D. R., Ellsworth, W. L., 2015b. Delayed dynamic triggering of deep tremor along the parkfield-cholame section of the san andreas fault following the 2014 m6. 0 south napa earthquake. Geophysical Research Letters 42 (19), 7916-7922.

Peterson, J., et al., 1993. Observations and modeling of seismic background noise. US Geological Survey Alburquerque, New Mexico.

Radiguet, M., Cotton, F., Vergnolle, M., Campillo, M., Walpersdorf, A., Cotte, N., Kostoglodov, V., 2012. Slow slip events and strain accumulation in the guerrero gap, mexico. Journal of Geophysical Research: Solid Earth 117 (B4).

Royer, A., Thomas, A., Bostock, M., 2015. Tidal modulation and triggering of low-frequency earthquakes in northern cascadia. Journal of Geophysical Research: Solid Earth 120 (1), 384-405.

Rubin, A. M., 2011. Designer friction laws for bimodal slow slip propagation speeds. Geochemistry, Geophysics, Geosystems 12 (4).

Rubin, A. M., Armbruster, J. G., 2013. Imaging slow slip fronts in cascadia with high precision cross-station tremor locations. Geochemistry, Geophysics, Geosystems 14 (12), 5371-5392. 
Ryberg, T., Haberland, C., Fuis, G., Ellsworth, W., Shelly, D., 2010. Locating non-volcanic tremor along the san andreas fault using a multiple array source imaging technique. Geophysical Journal International 183 (3), $1485-1500$.

Savard, G., Bostock, M. G., 2015. Detection and location of low-frequency earthquakes using cross-station correlation. Bulletin of the Seismological Society of America.

Schmidt, D., Gao, H., 2010. Source parameters and time-dependent slip distributions of slow slip events on the cascadia subduction zone from 1998 to 2008. Journal of Geophysical Research: Solid Earth 115 (B4).

Shelly, D. R., 2009. Possible deep fault slip preceding the 2004 parkfield earthquake, inferred from detailed observations of tectonic tremor. Geophysical Research Letters 36 (17).

Shelly, D. R., 2010. Migrating tremors illuminate complex deformation beneath the seismogenic san andreas fault. Nature 463 (7281), 648-652.

Shelly, D. R., Beroza, G. C., Ide, S., 2007a. Complex evolution of transient slip derived from precise tremor locations in western shikoku, japan. Geochemistry, Geophysics, Geosystems 8 (10).

Shelly, D. R., Beroza, G. C., Ide, S., 2007b. Non-volcanic tremor and lowfrequency earthquake swarms. Nature 446 (7133), 305-307.

Thomas, A. M., Beroza, G. C., Shelly, D. R., 2016. Constraints on the source parameters of low-frequency earthquakes on the san andreas fault. Geophysical Research Letters. 
Thomas, T. W., Vidale, J. E., Houston, H., Creager, K. C., Sweet, J. R., Ghosh, A., 2013. Evidence for tidal triggering of high-amplitude rapid tremor reversals and tremor streaks in northern cascadia. Geophysical Research Letters 40 (16), 4254-4259.

Ueno, T., Maeda, T., Obara, K., Asano, Y., Takeda, T., 2010. Migration of low-frequency tremors revealed from multiple-array analyses in western shikoku, japan. Journal of Geophysical Research: Solid Earth 115 (B9).

Wech, A. G., 2010. Interactive tremor monitoring. Seismological Research Letters 81 (4), 664-669.

Wech, A. G., Bartlow, N. M., 2014. Slip rate and tremor genesis in cascadia. Geophysical Research Letters 41 (2), 392-398.

Wech, A. G., Creager, K. C., 2008. Automated detection and location of cascadia tremor. Geophysical Research Letters 35 (20).

Yager, R. R., Filev, D. P., 1994. Generation of fuzzy rules by mountain clustering. Journal of Intelligent \& Fuzzy Systems 2 (3), 209-219. 


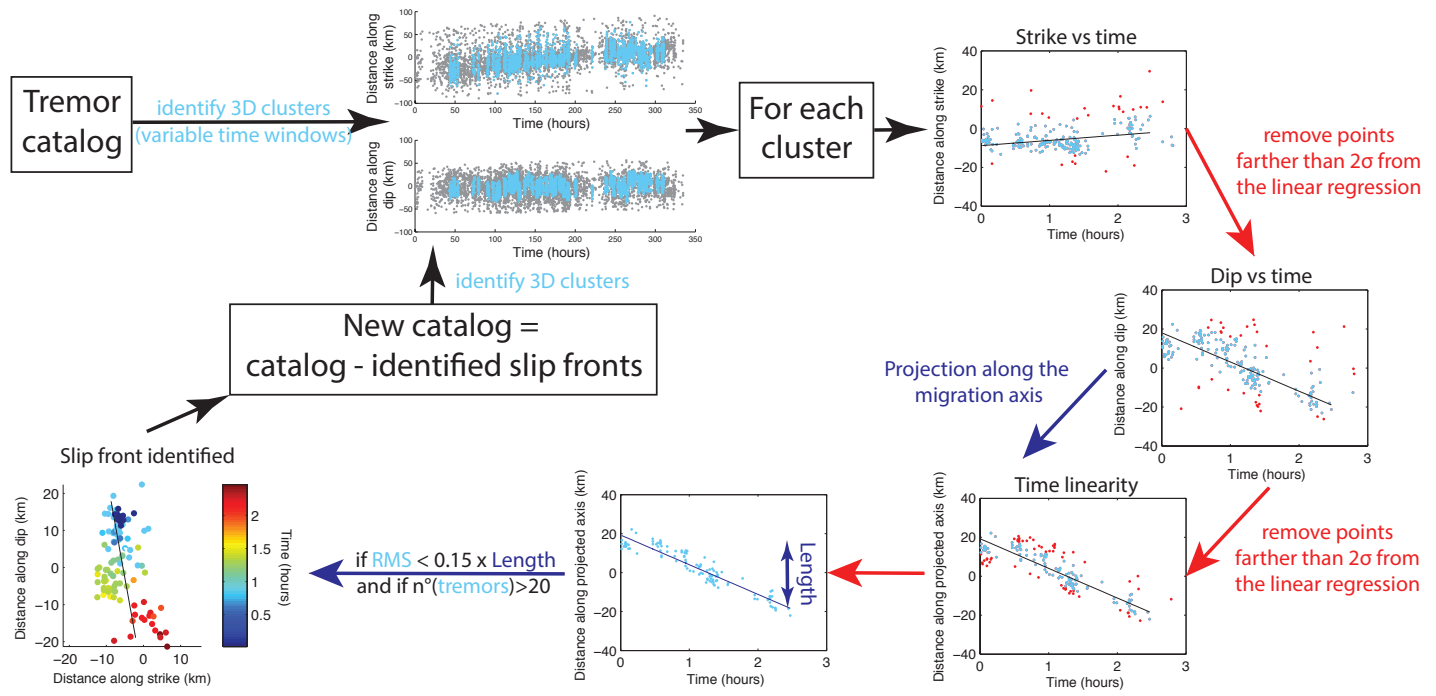

Figure 1: Algorithm scheme. See main text for description. 

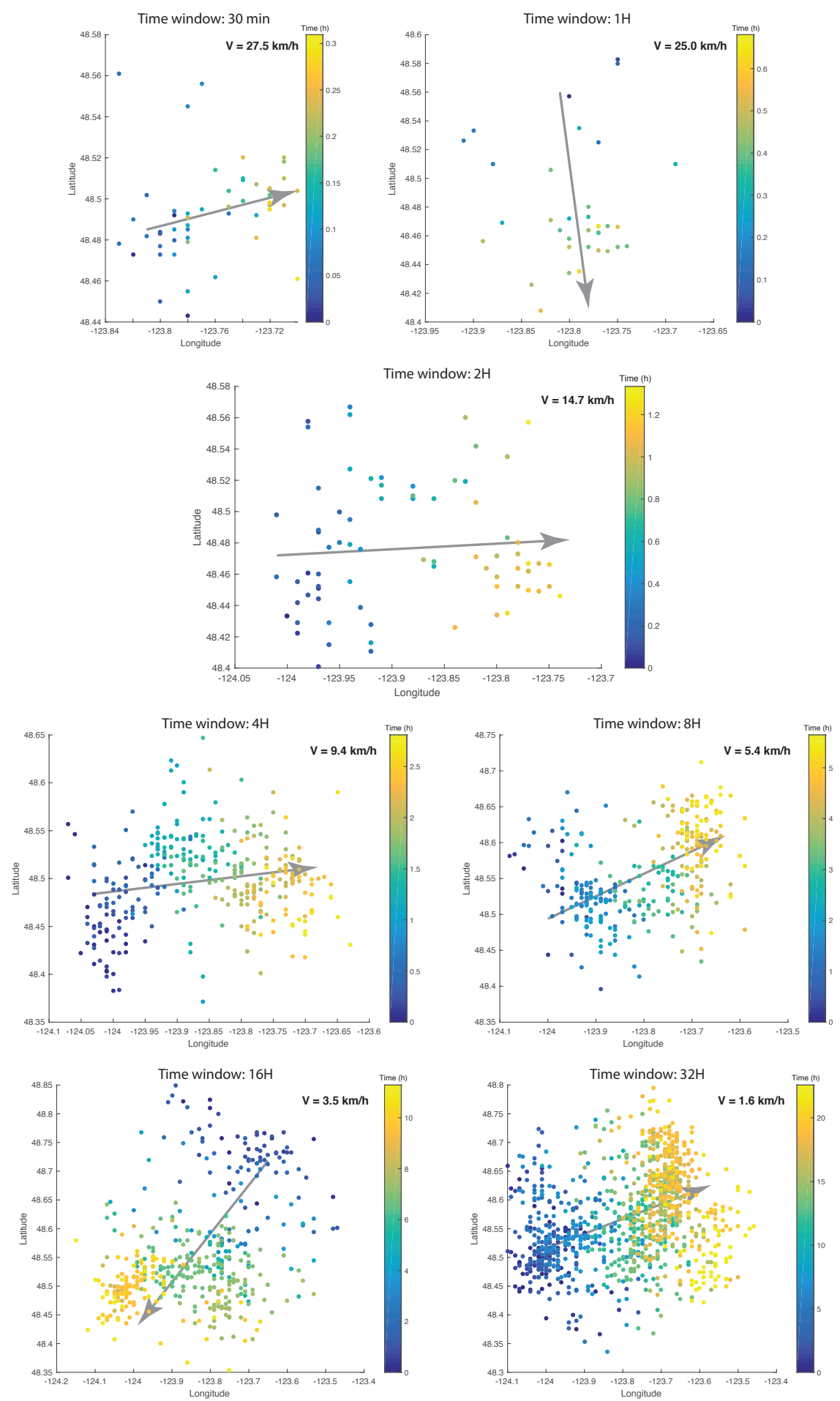

27

Figure 2: Examples of SSFs detected in the LFE catalog for the different time windows of our analysis. Grey arrows show SSF direction determined by the algorithm. 
Time window: $4 \mathrm{H}$

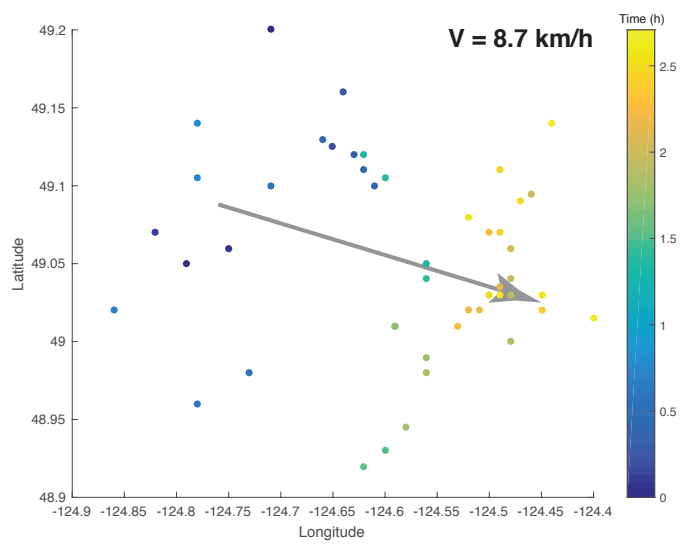

Time window: $16 \mathrm{H}$

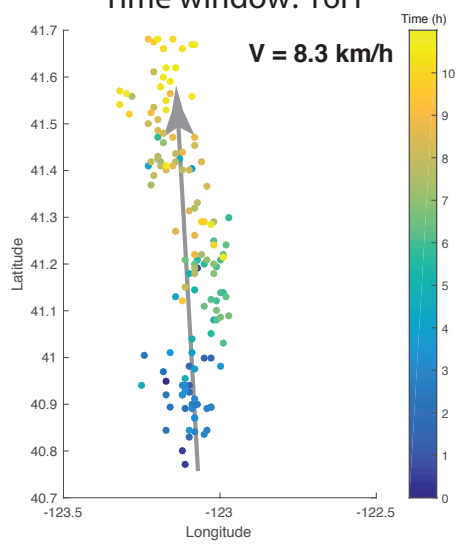

Time window: $8 \mathrm{H}$

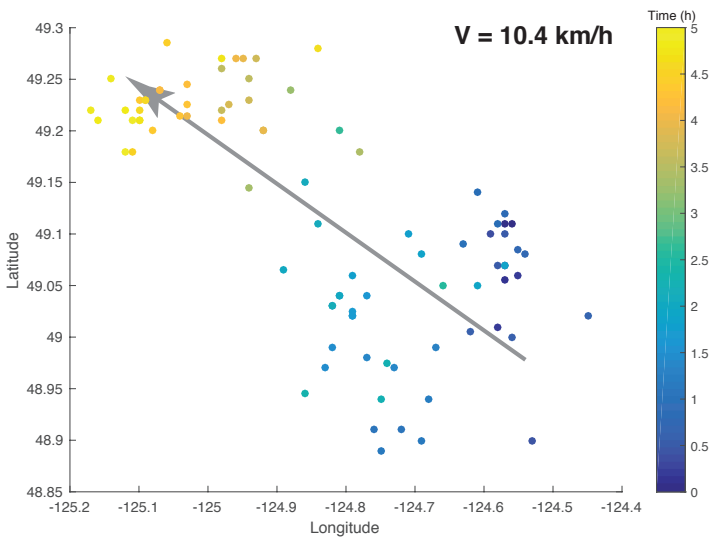

Time window: $32 \mathrm{H}$

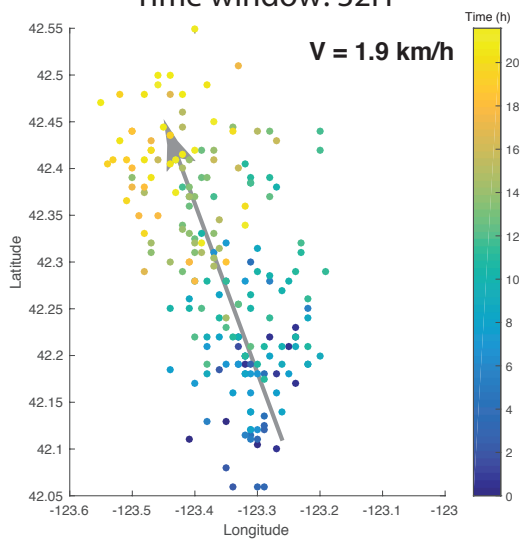

Figure 3: Examples of SSFs detected in the tremor catalog for the different time windows of our analysis. Grey arrows show SSF direction determined by the algorithm. 


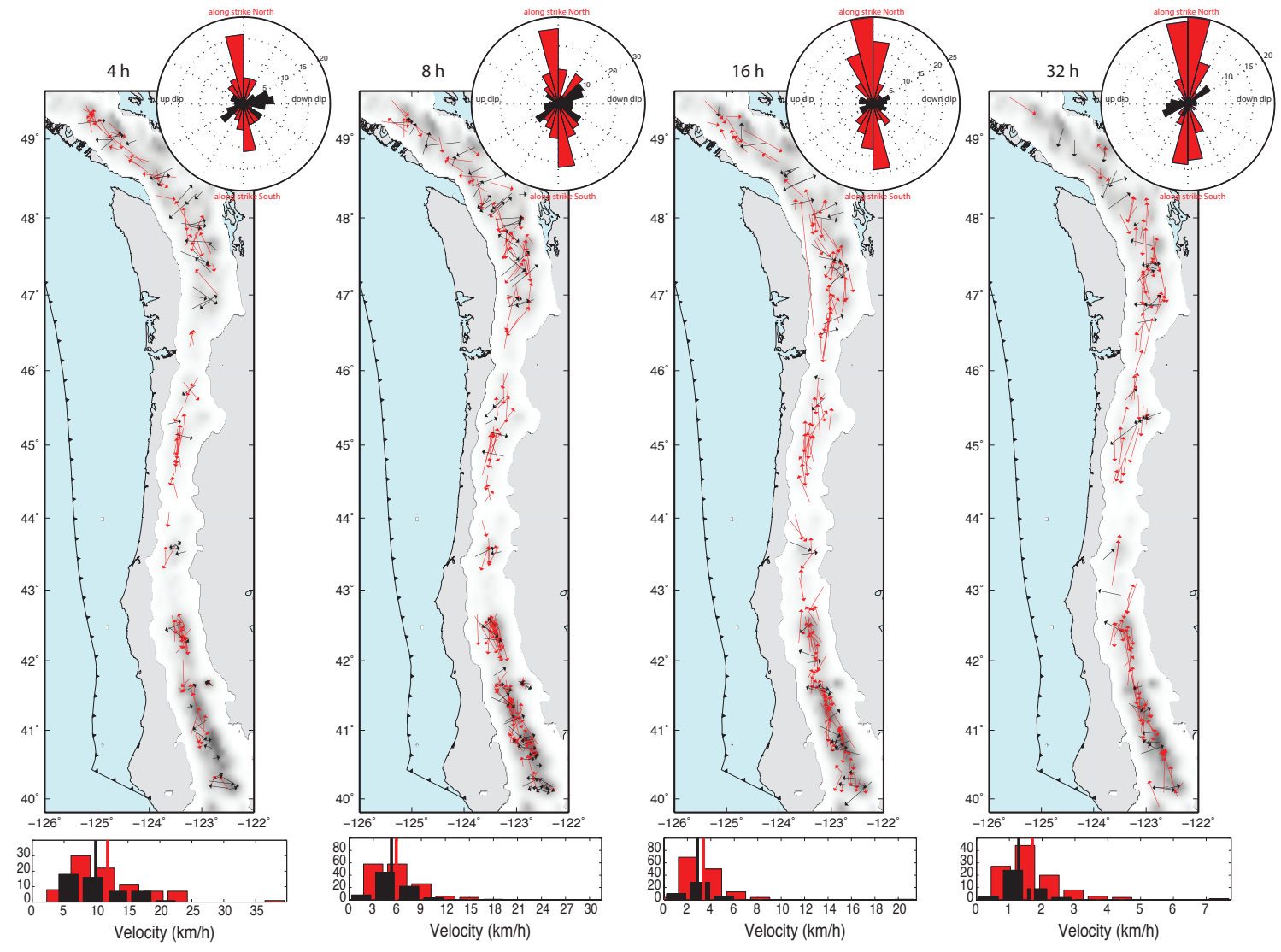

Figure 4: Detected secondary slip fronts in Cascadia from August 2009 to December 2015 for time windows of $4 \mathrm{~h}, 8 \mathrm{~h}, 16 \mathrm{~h}$ and $32 \mathrm{~h}$ (tremor catalog). White to gray color scale indicates the density of tremor detections. Arrows show lengths and directions of the SSFs propagating along strike (red) and along dip (black). Rose diagrams show the number of SSFs in the different directions. Histograms show velocity distributions for SSFs propagating along strike (red) and along dip (black). Red and black vertical lines superimposed on histograms show average velocity for SSFs propagating along strike and along dip, respectively. 


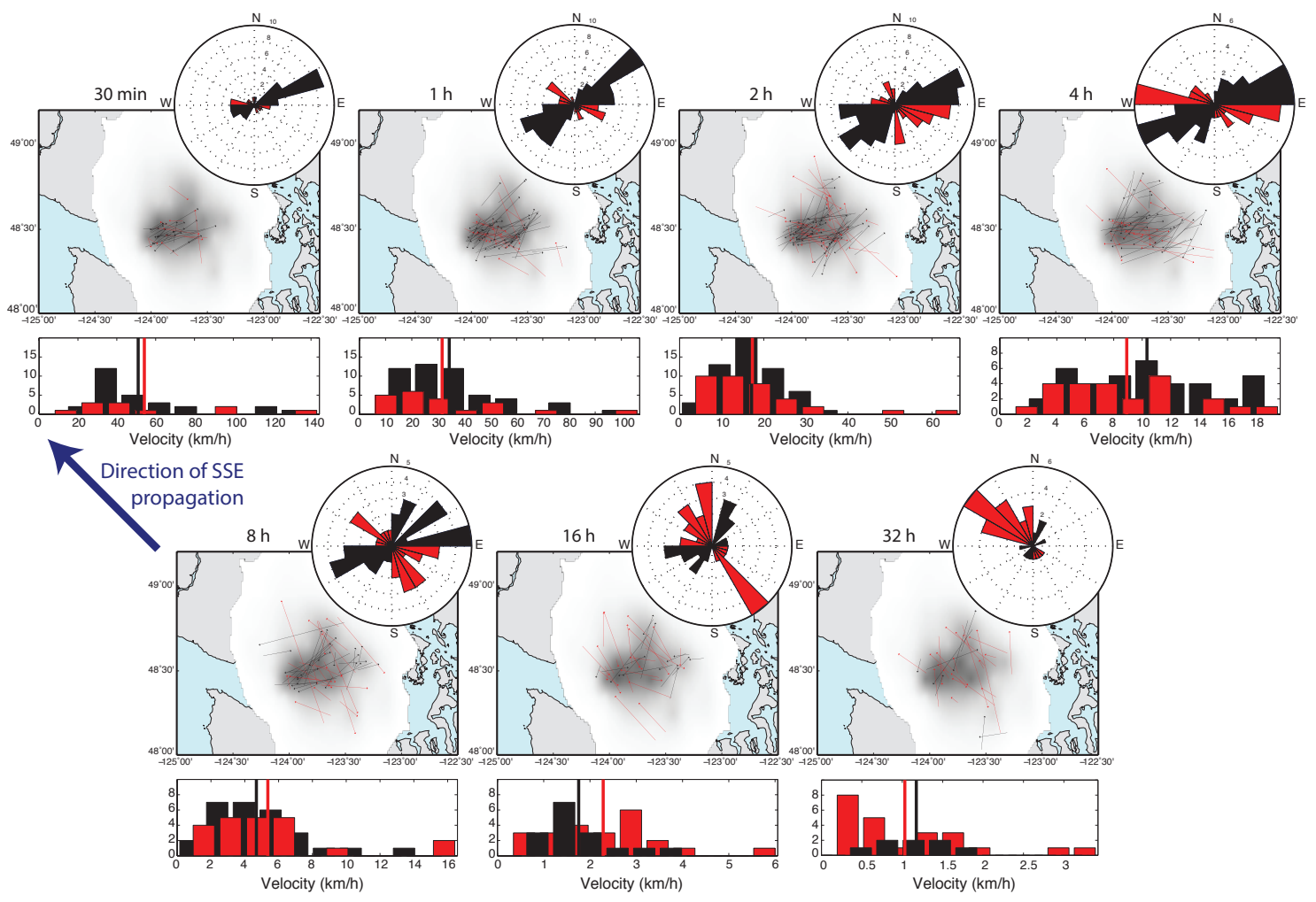

Figure 5: Detected secondary slip fronts during the 2003, 2004 and 2005 SSEs for time windows of $30 \mathrm{~min}, 1 \mathrm{~h}, 2, \mathrm{~h}, 4 \mathrm{~h}, 8 \mathrm{~h}, 16 \mathrm{~h}$ and $32 \mathrm{~h}$ (LFE catalog). White to gray color scale indicates the relative density of LFEs. Blue thick arrow show the approximate direction of the three SSEs ( $45^{\circ}$ west from north). Red arrows show lengths and directions of SSFs propagating in the direction of SSE propagation $\pm 45^{\circ}$ (along strike). Black arrows show lengths and directions of SSFs propagating perpendicular to the direction of SSE propagation $\pm 45^{\circ}$ (along dip). Rose diagrams show the number of SSFs in the different directions. Histograms show velocity distributions for SSFs propagating along strike (red) and along dip (black). Red and black vertical lines superimposed on histograms show average velocity for SSFs propagating along strike and along dip, respectively. 

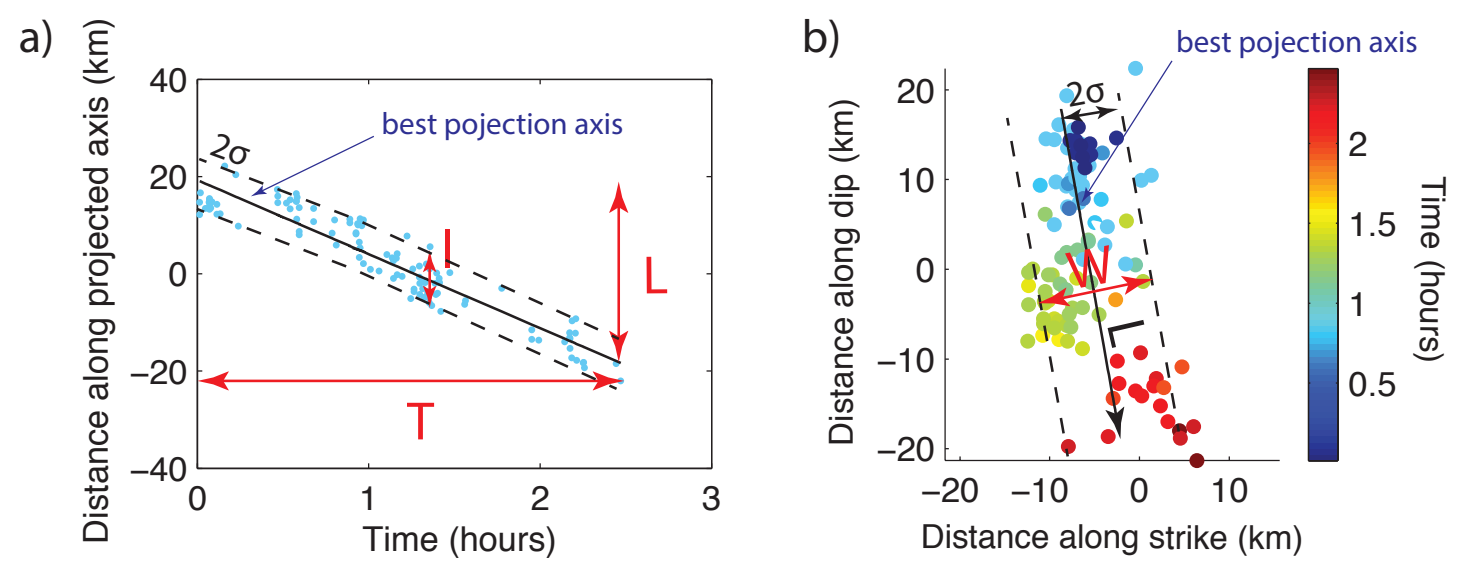

Figure 6: Scheme illustrating how SSF length $(L)$, width $(W)$, and pulse length $(l)$ are deduced from the analysis. $L$ is defined as the length of the best projection axis (parallel to the propagation direction) ( $\mathrm{a}$ and $\mathrm{b}$ ). $l$ is defined as the $2 \sigma$ dispersion from the linear regression of the distance along the best projection axis as a function of time (a). $W$ is defined as the $2 \sigma$ spatial dispersion (perpendicular to the propagation direction) from the best projection axis (b). 


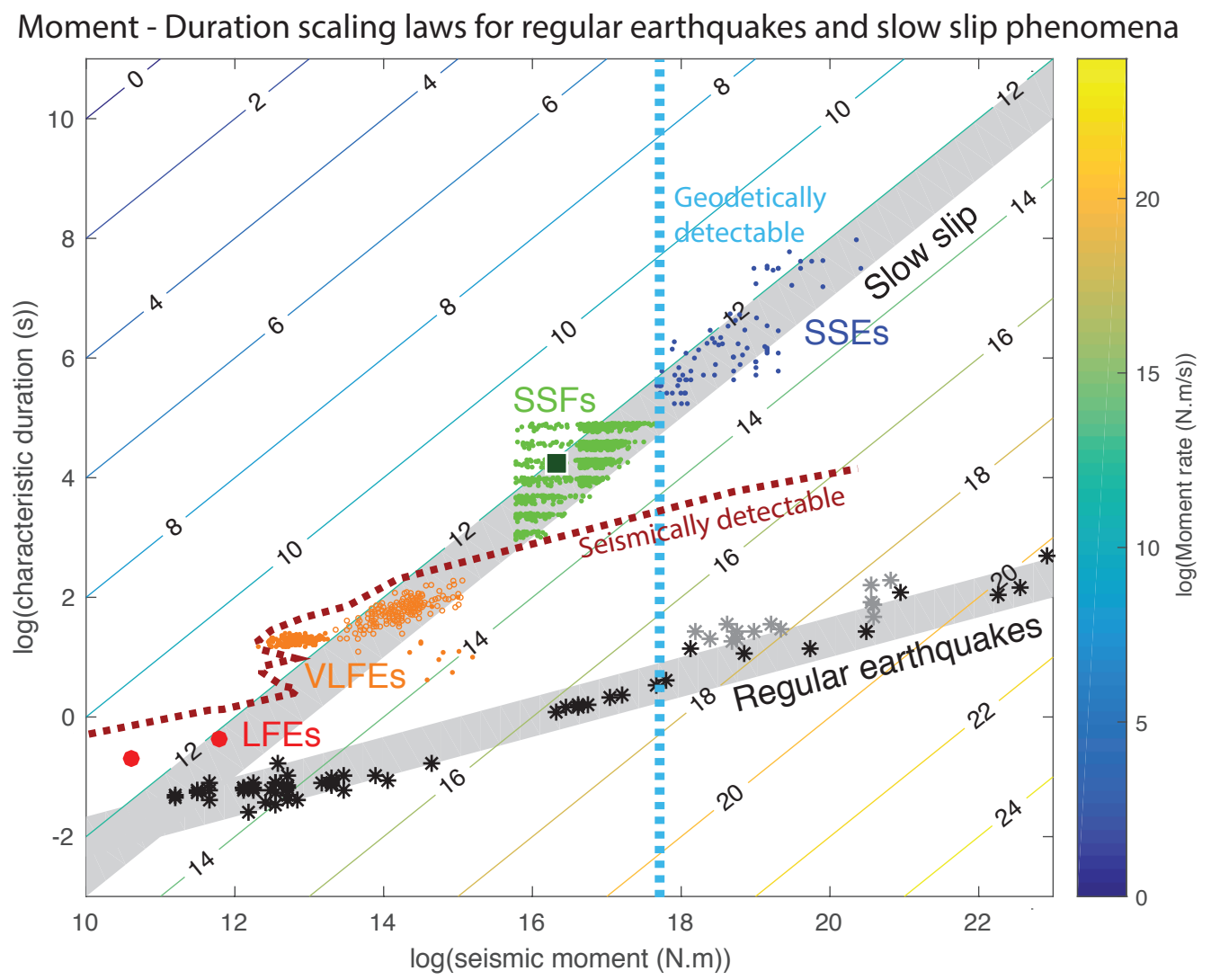

Figure 7: Moment - duration scaling law for regular earthquakes and slow slip phenomena (gray shaded areas). Colored solid lines are isolines of equal moment rate. Black stars indicate regular earthquakes over a large range of magnitude (compilation of Peng and Gomberg (2010) augmented with M4.8-5.8 events from the GCMT catalog and recent mega-earthquakes (Delouis et al., 2010; Bletery et al., 2014, 2016)). Gray stars indicate tsunami earthquakes (compilation from Peng and Gomberg (2010)). Blue dots are SSEs (compilation of Peng and Gomberg (2010) augmented with events from Gao et al. (2012) and Liu (2014)). Orange dots are VLFEs (Ito and Obara, 2006; Matsuzawa et al., 2009; Ide and Yabe, 2014). Orange circles are unnamed 20-200 s long duration earthquakes assimilated to VLFEs (Ide et al., 2008). Red circles are LFE moment and duration averaged over 100 (Thomas et al., 2016) and >100,000 events (Bostock et al., 2015). Cyan dashed line shows an approximate moment threshold $\left(M_{w} \approx 5.7-6.0\right)$ for geodetically detectable events. Red dashed line shows the limit of seismically detectable events (see text). In agreement with moment of large SSFs determined from strain meters (green square) (Hawthorne et al., 2016), SSFs detected in this study (green dots) span a moment - duration region that cannot be directly recorded either by common seismic or geodetic instrumentation. 